\title{
Influencia de las Instituciones de Educación Superior en el entorno urbano. Propuesta de análisis desde la perspectiva
} espacial

\section{Influência das instituições de ensino superior no meio urbano. Proposta de análise a partir da perspectiva espacial}

\author{
Influence of Higher Education Institutions in the urban \\ environment. Proposed analysis from the spatial perspective
}

\author{
Frida Landa Rivera \\ frida.landa@gmail.com \\ Universidad Autónoma de Ciudad Juárez - UACJ, México \\ Erick Sánchez Flores \\ esanchez@uacj.mx \\ Universidad Autónoma de Ciudad Juárez - UACJ, México
}

\begin{abstract}
Resumen: Un factor clave para entender la dinámica de desarrollo en las ciudades es la identificación del rol que juegan los diversos actores públicos y privados en la configuración de su estructura física, sociocultural y orientación productiva. En este sentido, las instituciones educativas, en particular las de educación superior como las universidades, pueden contribuir al desarrollo las ciudades en las que se localizan a través de las relaciones que generan con su entorno. Estas relaciones no siempre resultan evidentes y evaluar sus efectos pueden requerir enfoques complejos de análisis. En este trabajo se propone una aproximación para evaluar la forma en que las Instituciones de Educación Superior (IES) mexicanas contribuyen a configurar las condiciones de desarrollo urbano de sus ciudades sede, mediante un modelo cuantitativo bidimensional basado en un enfoque de análisis espacio-temporal. La dimensión espacial del análisis se utiliza para relacionar la presencia del campus universitario con características de la estructura urbana en el aspecto económico, de dinámica de uso de suelo y accesibilidad. El análisis incorpora variables de orden físico y socioeconómico en la búsqueda de patrones que revelen relaciones tangibles entre la universidad y la ciudad a escala intraurbana, en algunas de las principales ciudades mexicanas, exponiendo aquí los resultados de la aplicación del modelo en el caso de Ciudad Juárez, Chihuahua.
\end{abstract}

Palabras clave: configurador urbano, IES, universidad y ciudad.

Resumo: Um fator chave na compreensão da dinâmica do desenvolvimento nas cidades é a identificação do papel desempenhado pelos diversos atores públicos e privados na formação de sua orientação física, sociocultural e produtiva. Nesse sentido, as instituições educacionais, em particular as instituições de ensino superior, como as universidades, podem contribuir para o desenvolvimento de cidades em que estão localizadas através das relações que geram com seu meio ambiente. Essas relações nem sempre são óbvias e a avaliação de seus efeitos pode exigir abordagens complexas de análise. Este artigo propõe uma aproximação para avaliar a forma como as Instituições de Ensino Superior Mexicano (IES) contribuem para configurar as condições de desenvolvimento urbano de 
suas cidades-sede, através de um modelo quantitativo bidimensional baseado em uma abordagem de análise espaço-temporal. A dimensão espacial da análise é utilizada para relacionar a presença do campus universitário com características da estrutura urbana no aspecto econômico, a dinâmica de uso da terra e acessibilidade. A análise incorpora variáveis de natureza física e socioeconômica na busca de padrões que revelam relações tangíveis entre a universidade e a cidade em escala intra-urbana, em algumas das principais cidades mexicanas, explicando aqui os resultados da aplicação do modelo no caso de Ciudad Juárez, Chihuahua.

Palavras-chave: configurador urbano, IES, universidade e cidade.

Abstract: A key factor to understand the dynamics of development in cities is the identification of the role played by the various public and private actors in the configuration of its physical structure and sociocultural structures, and productive orientation. In this sense, educational institutions, particularly higher education institutions such as universities, can contribute to the development of cities in which they are located through the relationships they generate with their environment. These relationships are not always obvious and assessing their effects may require complex approaches to analysis. This paper proposes an approximation to evaluate the way in which the Mexican Higher Education Institutions (IES) contribute to configure the urban development conditions of its host cities, through a two-dimensional quantitative model based on a spatial-temporal analysis approach. The spatial dimension of the analysis was used to relate the presence of the university campus with characteristics of the urban structure in the economic aspect, the dynamics of land use and accessibility. The analysis incorporates variables of a physical and socioeconomic nature in the search for patterns that reveal tangible relations between the university and the city on an intra-urban scale, in some of the main Mexican cities, presenting results of the application of the model in the case of Ciudad Juárez, Chihuahua.

Keywords: urban configurator, IES, university and city.

\section{INTRODUCCIÓN}

La economía del conocimiento y la innovación social son algunas de las perspectivas teóricas que se han utilizado para entender la relación entre instituciones educativas y su entorno. Desde la primera, la universidad, como institución de educación superior (IES), destaca como elemento colaborador en la proyección regional de una ciudad al contribuir a la transformación de actividades económicas a través de la capacitación de profesionales capaces de obtener empleos especializados (BENNEWORTH, CHARLES; MADANIPOUR, 2010). El objetivo es sustituir la industria manufacturera y la exportación de recursos naturales por prestación de servicios. En su influencia a nivel urbano destaca la promoción de amenidades que vuelvan a la ciudad atractiva, integrando elementos de vivienda, accesibilidad y recreación, entre otros.

El acercamiento desde la innovación social evalúa la capacidad de las IES para provocar cambios que influyan en el desarrollo de la comunidad (BENNEWORTH; CUNHA, 2015). Se identifican programas educativos dirigidos a población vulnerable y se revisan 
las redes colaborativas entre las partes interesadas, como la comunidad universitaria -autoridades, maestros, alumnos-, empresarios, autoridades locales, organizaciones civiles y la misma población excluida a la que se dirigen (JULIANI et al., 2016).

El objetivo de la investigación es comprender la influencia de la universidad en la ciudad en la que se ubica, más allá de la difusión y extensión del conocimiento, a través del mejoramiento del entorno urbano. Desde 1929, las universidades mexicanas, tanto públicas como privadas, se convirtieron en los capacitadores para cubrir la demanda de empleados de las empresas (LOREY, 1994). La educación superior se diversifica en otro tipo de instituciones que varían en su misión y objetivos, por lo que el concepto de IES resulta el más adecuado para incluir los casos de estudio analizados en este caso.

Se presume que identificar los patrones de concentración o dispersión de ciertos rasgos urbanos, asociados a la presencia de las IES, permite identificar su nivel de influencia en el entorno urbano cercano a sus instalaciones físicas, denominadas campus. En este caso, el concepto de distancia se utiliza para inferir dicho grado de influencia. Además, las IES pueden jugar un papel central en la atracción de capital humano que resulta importante para la vitalidad económica de la ciudad, que se complementa con atributos urbanos como la diversidad, la promoción de la escala humana y la accesibilidad (DEN HEIJER, 2008). Por lo tanto, es necesario delinear la metodología que hará posible asignar valores a la influencia, cuya posterior interpretación permita identificar cualidades positivas o negativas para el entorno.

Las características urbanas asociadas la presencia de la IES serán equivalentes a las variables de la investigación. La metodología incluye tres esferas: 1. Económica, 2. Dinámica de suelo y 3 . Accesibilidad. A partir de estas se derivan siete variables presentes en el análisis espacial (Fig. 1).

Figura 1. Dimensión horizontal y vertical de la estrategia metodológica.

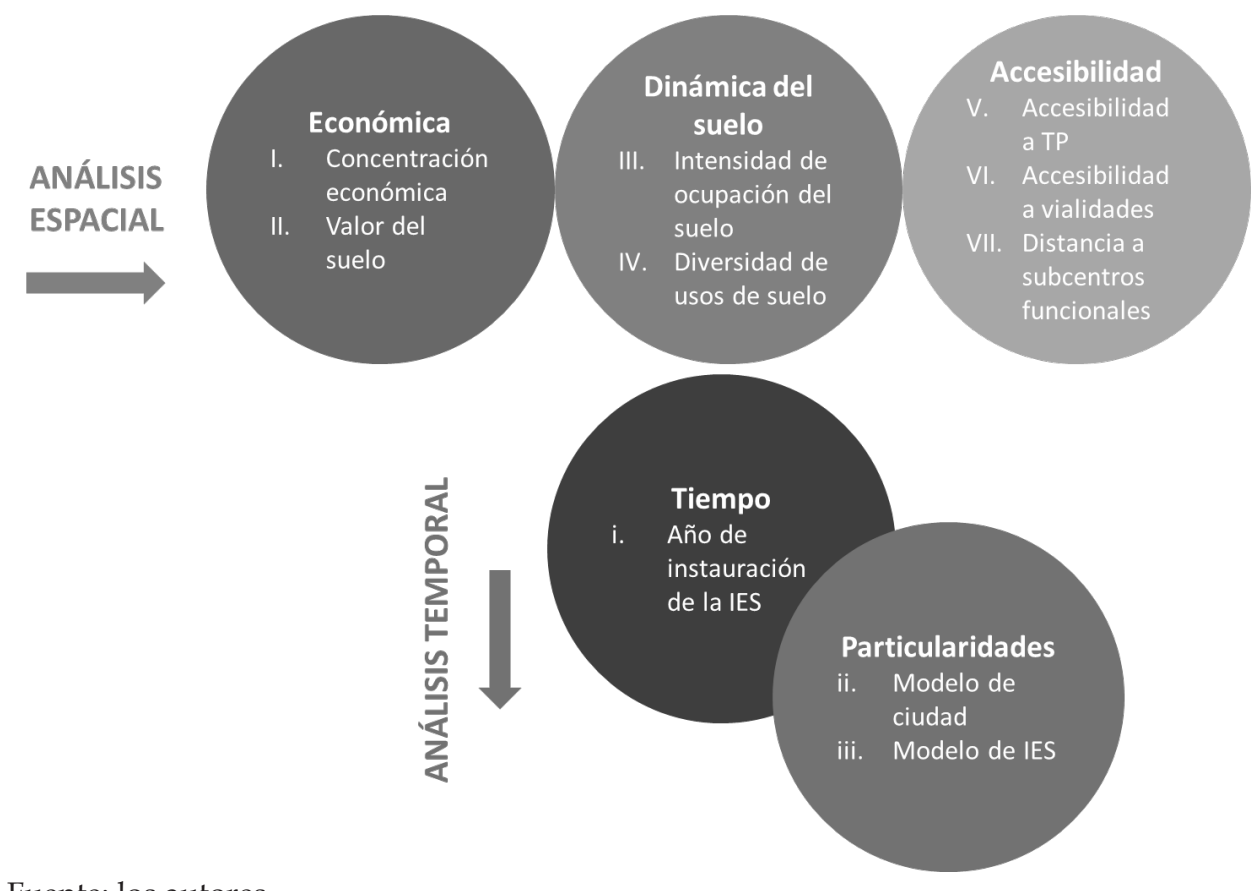

Fuente: los autores. 
La primera esfera incluye las variables de concentración de actividades económicas y de valor del suelo. El primer caso, constituye una de las formas más directas de observar la posible atracción, sobre todo de actividades de servicio y comercio, al concentrarse en el campus un mercado "cautivo" que incrementa la rentabilidad de las actividades que ocurren en su entorno. La segunda variable es referida como la forma más rápida de obtener el valor económico que le genera la universidad a la ciudad, ya que la influencia en la economía local y regional sería muy difícil de calcular. La parte crítica de este elemento corresponde al impacto que el aumento de los valores de suelo provocaría en la comunidad adyacente al campus (DEN HEIJER, 2008).

La segunda esfera que se refiere al impacto en la dinámica del suelo se mide a través de la influencia del campus sobre la diversidad de los usos de suelo y la intensidad de ocupación del mismo. Algunos autores sugieren que la diversidad emana del aumento en la demanda de servicios por parte de la comunidad universitaria. Se ha mencionado el desplazamiento de la población original debido al aumento de las rentas. Por el contrario, algunas autoridades locales intentan beneficiarse del incremento de amenidades que puedan generar beneficios para la comunidad que, si bien son económicos, también se relacionan con el aumento del atractivo del ambiente urbano (RUSSO, VAN DEN BERG; LAVANGA, 2004; FERNÁNDEZ-ESQUINAS; PINTO, 2014).

La tercera esfera correspondiente a la accesibilidad se relaciona con el número de alternativas para realizar actividades. A mayor número de opciones se considera que existe una mejor accesibilidad. Por lo tanto, se derivan tres variables, las dos primeras cuantifican las opciones disponibles para transporte público y vialidades principales que reflejan la facilidad o dificultad de traslado desde y hacia el campus. La tercera mide la distancia de la IES hacia el centro o subcentro urbano más cercano, suponiendo que estas zonas concentran servicios, comercio y por lo tanto empleo, se relaciona con alternativas de actividades accesibles a distancias cortas del campus.

La hipótesis de la investigación plantea que las IES actúan como configuradores urbanos cuya influencia refleja a través de patrones espaciales derivados de la presencia de su campus. Esta influencia tiene entonces una primera manifestación espacial a través de siete variables medidas en el estudio: Concentración económica, Valor de suelo, Intensidad de ocupación, Diversidad de usos de suelo, Accesibilidad al transporte público, a las Vialidades y la Distancia a centros funcionales. La intensidad de la manifestación espacial depende proporcionalmente del tiempo que tiene establecida la IES, además de presentar particularidades según el modelo de ciudad y los modelos administrativo y educativo de la IES.

\section{EL ANÁLISIS DEL ESPACIO PARA ENTENDER LOS CAMBIOS URBANOS}

La propuesta de análisis de la relación espacial entre la universidad y la ciudad se apoya en un enfoque positivista basado en un modelo deductivo-nomológico de Hempel y Oppenheim en 1958. En este modelo se explica un suceso a través de un proceso empírico que permita declarar leyes y las circunstancias en las que éstas aplican (PEET, 1998). Autores 
como Peter Hagget, fueron quienes iniciaron el tratamiento del espacio dentro del método hipotético-deductivo para, a través de su descripción, construir teoría que permita explicar las relaciones establecidas entre la sociedad y su medio ambiente (HUBBARD, KITCHIN; VALENTINE, 2008).

Este enfoque permite introducir el componente espacial a la explicación de patrones percibidos en la evolución de las ciudades. La adición del tiempo ayuda a entender el proceso de influencia de los procesos económicos y sociales en el espacio y también del espacio hacia los procesos (HAINING, 2003). Las investigaciones que incorporan la modelación y la cuantificación de variables retoman importancia con la evolución en herramientas como los Sistemas de información geográfica (SIG) que permiten, a través del análisis espacial, percibir una parte de la construcción social del espacio a través de los procesos de cambio (HUBBARD; KITCHIN; VALENTINE, 2008).

\section{SELECCIÓN DE CASOS DE ESTUDIO}

La selección de los casos de estudio surgió de las 30 zonas metropolitanas (ZM) con más de 500 mil habitantes propuestas por el Consejo Nacional de Población (CONAPO, 2012). Dentro del grupo de una $Z M$, que puede incluir municipios y localidades, se tomó en cuenta la localidad principal. La ZM del Valle de México presenta una diferencia de población notoria con el resto del país por lo que se descartó del estudio. La imposibilidad de obtener información de las variables de una ciudad fue motivo de eliminación. Tal fue el caso de Poza Rica, Oaxaca y Pachuca, las dos primeras no tienen carta urbana disponible, mientras que Poza Rica ni Pachuca otorgan información sobre las rutas de transporte público (Fig. 2).

Figura 2. ZM propuestas como casos de estudio.

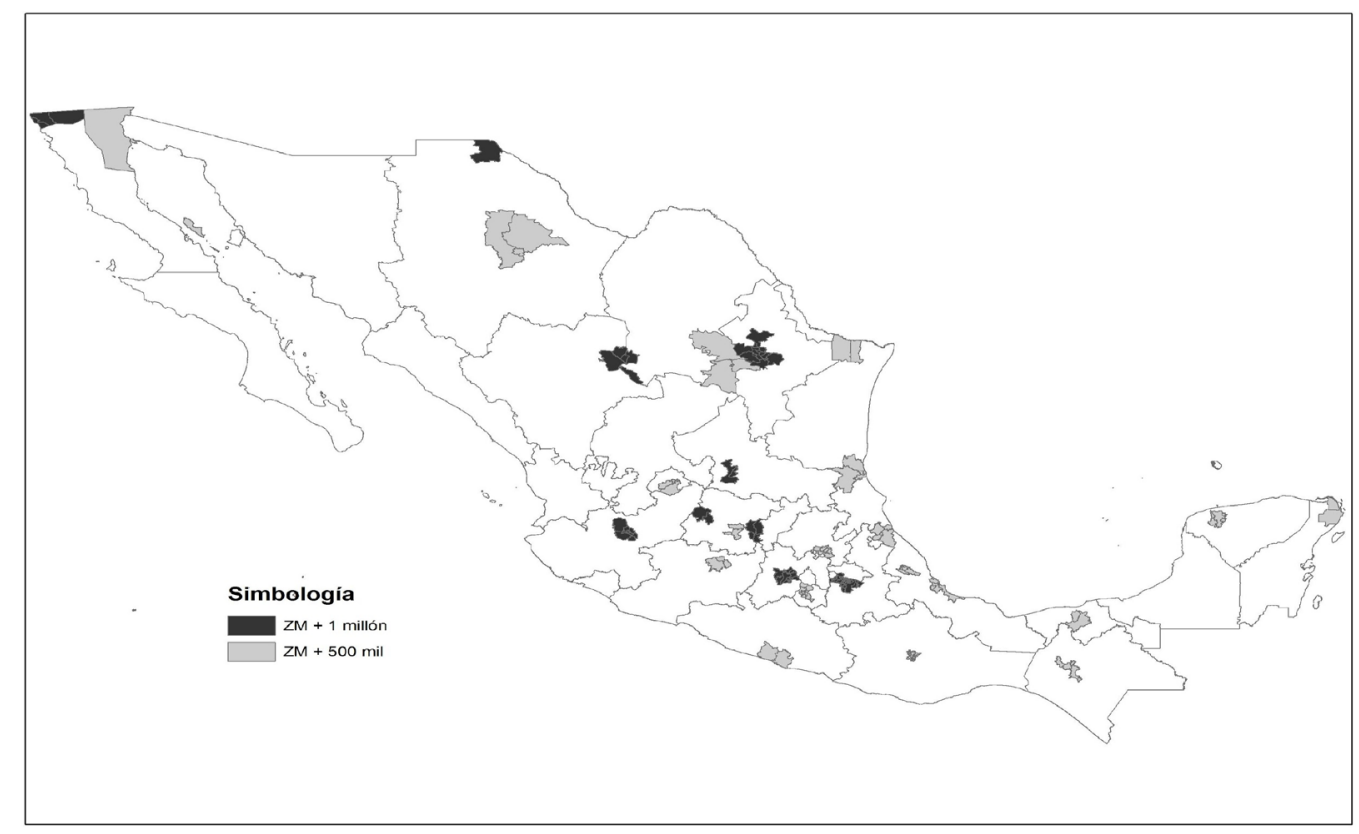

Fuente: Elaborado a partir del Sistema Urbano Nacional (SUN) del Consejo Nacional de Población (CONAPO, 2012) 


\section{ESTRATEGIA METODOLÓGICA DE LA DIMENSIÓN HORIZONTAL}

El análisis espacial puede definirse como una colección de técnicas metodológicas para entender las relaciones de los fenómenos que ocurren teniendo al espacio como marco de referencia. Haining (2003) menciona tres componentes del análisis espacial: 1) el modelado cartográfico, que implica la representación en mapas de un conjunto de datos; 2) el modelado matemático, resultado de la interacción espacial entre los objetos cuyos valores fueron introducidos y su posición geográfica; y 3) el desarrollo y aplicación de técnicas estadísticas.

La selección de la información y la escala aplicada en el modelo, entre otros factores, influyen en los resultados obtenidos, por lo que la dependencia espacial que pueda encontrarse deberá ser revisada bajo estas consideraciones (HAINING, 2003). Las investigaciones que incluyan el método inductivo deberán ser cuidadosas al hacer afirmaciones, especificando el contexto en el que pueden suceder las predicciones.

La manifestación espacial de la IES se midió a partir de la identificación de los patrones espaciales de las siete variables que se eligieron para identificar la influencia de la universidad con su entorno inmediato. Una relación espacial positiva identifica a la IES como un dinamizador urbano, mientras que una relación negativa se refiere el papel de la IES como inhibidor de los atributos medidos. Un patrón aleatorio señalaría la inexistencia de una organización espacial perceptible. La dimensión vertical implicaría la comparación de los atributos de varios casos de estudio en una escala temporal, sirviendo de apoyo a la primera parte del análisis.

Los siguientes apartados incluyen los datos y las fuentes necesarias para la construcción de las variables. Se requirió asociar los datos a una unidad espacial que permitiera homogeneizar la información que de lo contrario sería imposible comparar. La unidad espacial utilizada fue el Área Geoestadística Básica (AGEB) utilizada por el Instituto Nacional de Estadística, Geografía e Informática (INEGI), misma que ayudó a delimitar el área de estudio para cada ciudad, utilizando el contorno de la superficie con AGEBs disponibles.

\section{Concentración económica}

La variable se conforma seleccionando las amenidades que podrían atraerse y concentrarse en relación a una IES. En el caso de las ciudades mexicanas se recurrió al Directorio Estadístico Nacional de Unidades Económicas (DENUE) extrayéndose cinco tipos de negocios. Los datos se obtuvieron a través del portal en internet del instituto, el formato de los archivos es compatible con SIG. La representación es a través de puntos georreferenciados asociados a una tabla de contenido con información concerniente al levantamiento del Censo Económico finalizado en 2014 y publicado en 2015.

Los establecimientos fueron: 1. Cafeterías; 2. Restaurantes; 3 . Alimentos de consumo inmediato; 4. Papelerías, incluyendo solamente la venta de artículos al por menor; 5 . 
Minisúper, seleccionando los establecimientos menores a $600 \mathrm{~m}^{2}$. Los establecimientos fueron agregados a través de su ubicación dentro del polígono perteneciente a una AGEB.

\section{Valor del suelo}

El valor del suelo se identifica de acuerdo con el precio en pesos (MXN) por metro cuadrado para cada zona que haya establecido la Dirección de Catastro en una ciudad. Este precio se obtuvo a partir de las tablas de valores catastrales por municipio correspondientes para el año 2017, o en su defecto el más reciente disponible, publicadas en los periódicos oficiales de cada entidad federativa. La información que no estuvo accesible directamente tuvo que ser solicitada a través de los sistemas INFOMEX de cada estado, de acuerdo a la Plataforma Nacional de Transparencia. Las tablas, sin embargo, no tienen un formato estándar, los valores se obtuvieron por colonia o por distritos y sectores catastrales. Al ser representados por polígonos distintos a los límites establecidos por las AGEB, fue aplicado un procedimiento estadístico zonal que permitió la asociación con la unidad homogeneizadora.

\section{Intensidad de ocupación}

La variable se calcula a través del coeficiente resultante de dividir el área ocupada por un uso entre el área total de cada AGEB. El área ocupada se obtuvo previamente al restarle a la superficie total de la unidad espacial homogeneizadora la superficie no construida. La obtención de la superficie no construida se hizo a través de percepción remota utilizando imágenes satelitales Landsat 8 disponibles en el sistema GloVis (Global Visualization Viewer) de la agencia estadounidense United States Geological Survey (USGS). A estas imágenes se les aplicó una clasificación supervisada a través un software especializado, que consiste en elegir pixeles similares para asignarles una categoría, en este caso se crearon dos: construido y no construido. La imagen satelital es analizada con la información otorgada en el paso anterior y se obtiene la clasificación de la imagen completa para cada ciudad y se cuantifica el porcentaje de superficie construida por AGEB.

\section{Diversidad de usos de suelo}

La diversidad de uso se designará con la medida de uniformidad asociado al Índice de Shannon-Wiener (McGARIGAL; MARKS, 1995). Este índice es una medida matemática que surge de la teoría de la información desarrollada en 1949, aplicándose para medir la diversidad de información contenida en un código o mensaje. Uno de los usos del índice en temas urbanos implica la evaluación del aprovechamiento del suelo. Se considera que la diversidad es mayor cuando existe una alta presencia de usos diversos o mixtos, que se 
contrapongan al uso monofuncional en un sector de la ciudad ya sea a escala de manzana, barrio, entre otras (GARCÍA; GARCÍA, 2005).

La información de la carta urbana se agrega a la unidad espacial para obtener los usos de suelo contenidos en cada una. Este procedimiento permitirá obtener el área de cada tipo de uso y su proporción con el área total. A partir de estos dos datos será posible aplicar las fórmulas para el índice de Shannon y su medida de uniformidad, que representa un valor de diversidad de uso de suelo para cada AGEB.

\section{Accesibilidad al transporte público}

Los datos requeridos para construir la variable son las rutas de transporte, de las que se obtienen las intersecciones. El proceso necesita realizar un conteo aditivo de los nodos por cada AGEB. Las rutas de transporte público, a excepción de Ciudad Juárez y Chihuahua que fueron obtenidas directamente en formato shapefile, se consiguieron en archivos PDF que fueron convertidos a imágenes para georeferenciar en SIG, sirviendo para el trazado de cada ruta. En otros casos fue necesario recurrir a páginas online donde se consultaron las rutas de manera individual y así poder mapear la información obtenida.

\section{Accesibilidad a vialidades}

La variable se construye de forma similar a la anterior, sustituyendo las rutas de transporte público por las vialidades principales, de las que también se obtuvieron los nodos que se agruparon por AGEB. Se obtuvo el archivo de ejes viales del INEGI. A partir del archivo original, disponible por estado, fue necesario seleccionar únicamente la superficie de estudio, correspondiente al contorno que conforman los AGEBs disponibles para la ciudad. Posteriormente se eligieron aquellos ejes pertenecientes al tipo vial de Avenida, con el fin de encontrar las intersecciones entre las calles principales.

\section{Distancia promedio a subcentros funcionales}

Se obtuvieron los centros y subcentros propuestos en las cartas urbanas de cada ciudad. A pesar de que se encuentran marcados por los Ayuntamientos como sitios óptimos, los planes de los que se derivan señalan que no siempre están consolidados pero se espera que en un futuro se comporten como tales. El procedimiento para la captura fue el mismo que el aplicado para los usos de suelo, ubicando los puntos con base en la carta urbana.

\section{Índices de autocorrelación espacial}

Getis y Ord (1992) recomiendan el uso en conjunto de estadísticas G e I para identificar patrones locales que no podrían detectarse a nivel global. Por lo tanto, se utilizará el 
índice de Moran (Moran's I) como medida de autocorrelación que compara la media de un conjunto de datos para analizar si existe agrupación de valores similares, sin especificar la presencia de valores bajos o altos. El otro índice utilizado es el Gi* de Getis-Ord que identifica zonas críticas donde se aglomeran valores altos o valores bajos, a las que se les denomina hot spots y cold spots respectivamente. La proximidad de valores que tomará el SIG para calcular ambos índices puede establecerse través de los vecinos inmediatos o especificando una distancia determinada. En este caso se establecerá una banda de distancia con valor de 500 metros.

Posteriormente a la aplicación de los índices, los valores promedio por AGEB de cada variable serán promediadas en cuatro radios de influencia, a 500, 1000, 1500 y 2000 metros. El objetivo es advertir si a mayor cercanía del campus existen valores más altos que representan una mayor evidencia de su influencia, a excepción de la distancia a subcentros funcionales, ya que los valores más bajos demuestran mayor cercanía a éstos.

\section{RESULTADOS EN CIUDAD JUÁREZ}

Se identifican cuatro de las principales instituciones de la ciudad, representadas por seis campus, que surgen a partir del crecimiento poblacional y la especialización industrial, que hicieron necesario cualificar a la población según la demanda de empleos: El Tecnológico de Ciudad Juárez (ITCJ), la Universidad Autónoma de Chihuahua (UACH), la Universidad Autónoma de Ciudad Juárez con los campus correspondientes al Instituto de Ciencias Sociales y Administración (ICSA), el Instituto de Ciencias Bioquímicas (ICB) y el que integra al Instituto de Arquitectura, Diseño y Arte y el Instituto de Ingeniería y Tecnología (IADA-IIT).

\section{Concentración económica}

Los hot spots de concentración económica coinciden solamente con el campus de ITCJ. Mientras que los valores de suelo se concentran en la zona norte de la ciudad, donde se encuentran todos las IES propuestas pero ninguna AGEB considerada como hot spot coincide en alguna donde se localice un campus. En ambos casos no existen cold spots detectados (Fig. 3). 
Figura 3. Aplicación del índice Gi* de Getis-Ord para la esfera económica.
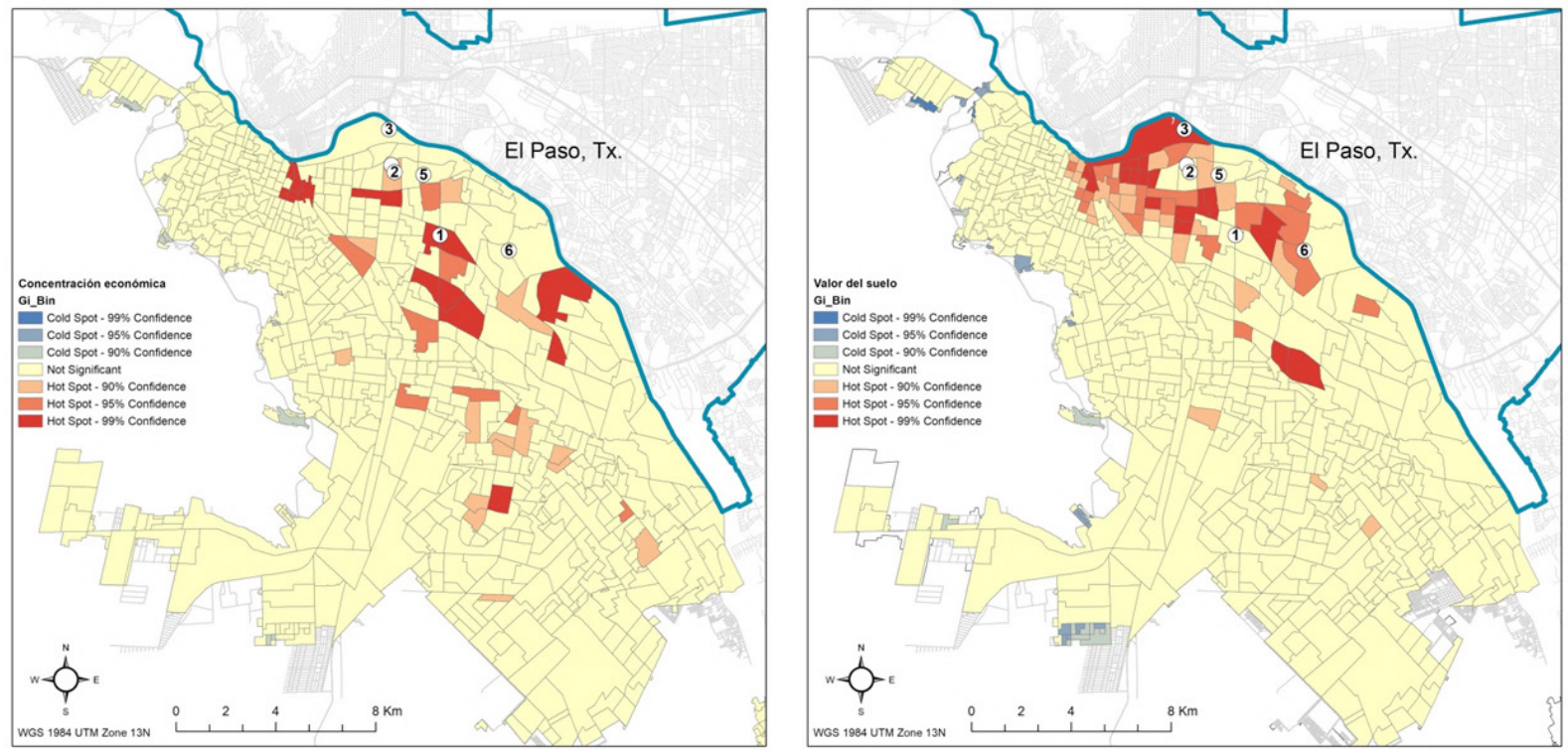

1: ITCJ; 2: UACH; 3: ICSA; 4: ICB; 5: IADA/ITT; 6: ITESM

Fuente: los autores.

La primera variable muestra que solamente en el caso de ITCJ, la UACH y IADAITT existe mayor concentración de establecimientos asociados a la presencia del campus en la banda de distancia a 500 metros. ITESM e ICSA por el contrario, presentan menos establecimientos cercanos al campus, que van aumentando conforme se alejan de estos. El caso de ICB muestra un patrón aleatorio. El valor de suelo en ITCJ, UACH e ICB incrementa a mayor distancia del campus. El único caso en que se muestra un aumento del valor a menor distancia es ICSA. ITESM y IADA-ITT tienen valores más altos a una distancia de 1000 y 1500 m que a 500 m, y disminuyen con mayor evidencia a partir de los 2000 m (Fig. 4).

Figura 4. Variables de la esfera de concentración económica a distintas bandas de distancia.
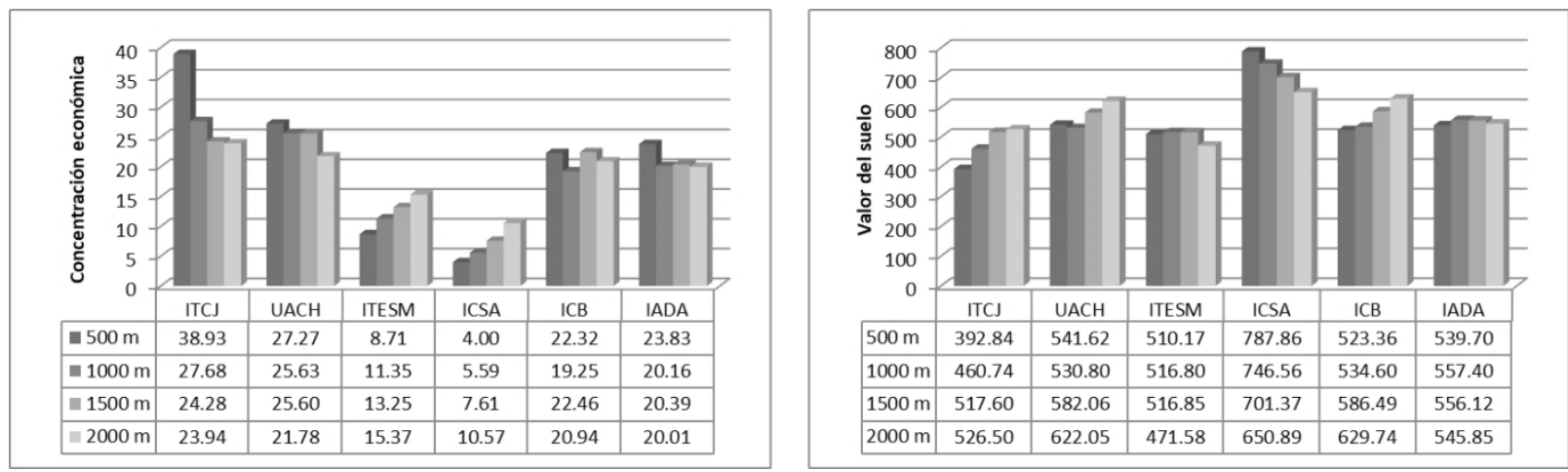

Fuente: los autores. 


\section{Dinámica del suelo}

Las dos variables carecen de hot spots, es decir, no hay una AGEB de valores altos rodeada por similares. Por lo tanto, no se puede deducir que los campus atraigan mayor ocupación de suelo o variedad de usos. La asociación de valores bajos tampoco se localiza en la cercanía de las IES (Fig. 5).

Figura 5. Variables de la esfera de ocupación de suelo o variedad de usos.
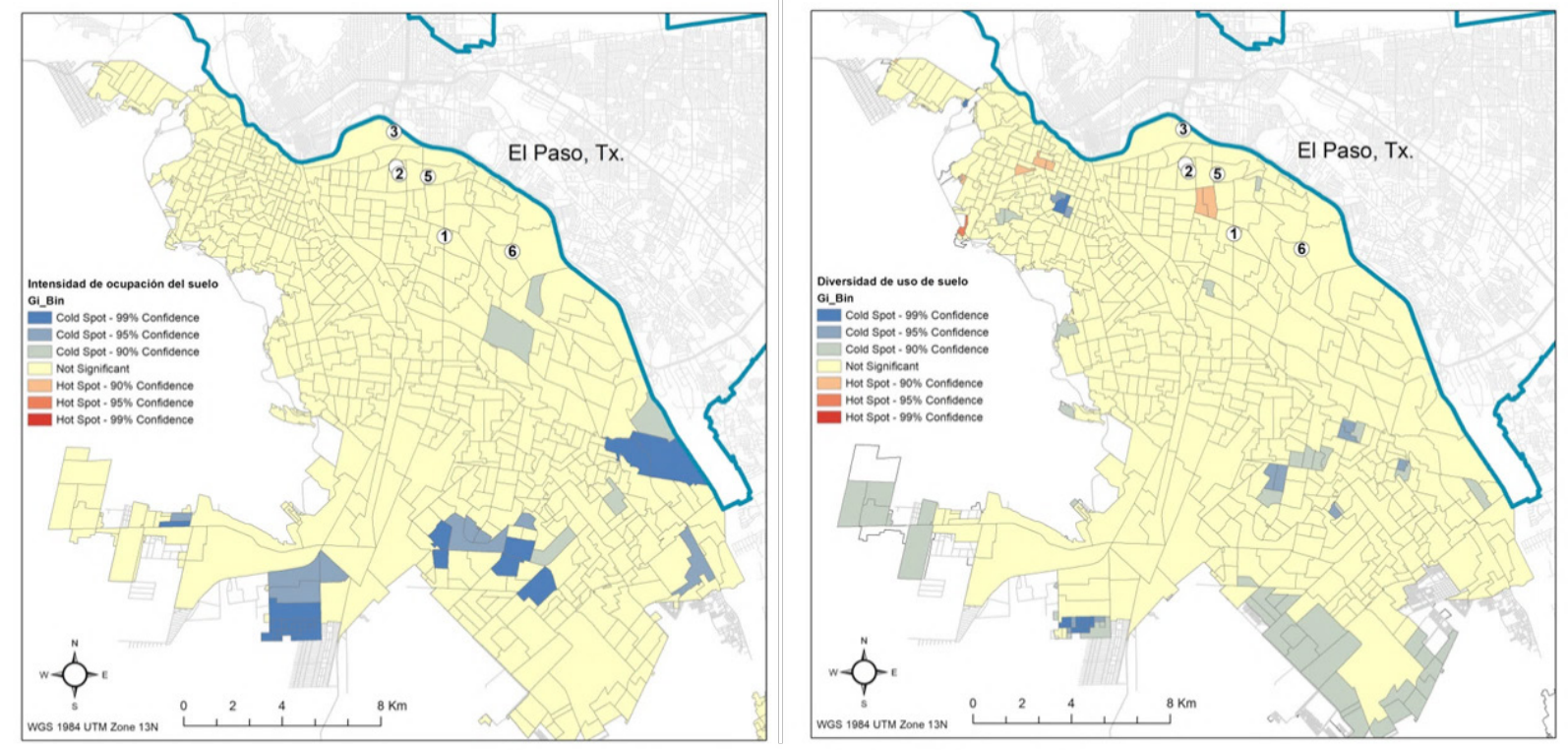

1: ITCJ; 2: UACH; 3: ICSA; 4: ICB; 5: IADA/ITT; 6: ITESM

Fuente: los autores.

Todos los campus muestran valores altos en la intensidad de ocupación de suelo. Solamente en el caso del ITESM se observan valores menores a menor distancia. La diversidad de usos de suelo aumenta a menor distancia en los casos de la UACH e ICB. $\mathrm{Al}$ contrario, disminuyen en ICSA e ITCJ. Los valores en ITESM son similares en todas las bandas, con variaciones entre el mayor y menor de 0.04. IADA-ITT también presenta valores similares, ligeramente mayores a 1000 y 1500 m (Fig. 6).

Figura 6. Variables de la esfera de dinámica de suelo a distintas bandas de distancia.
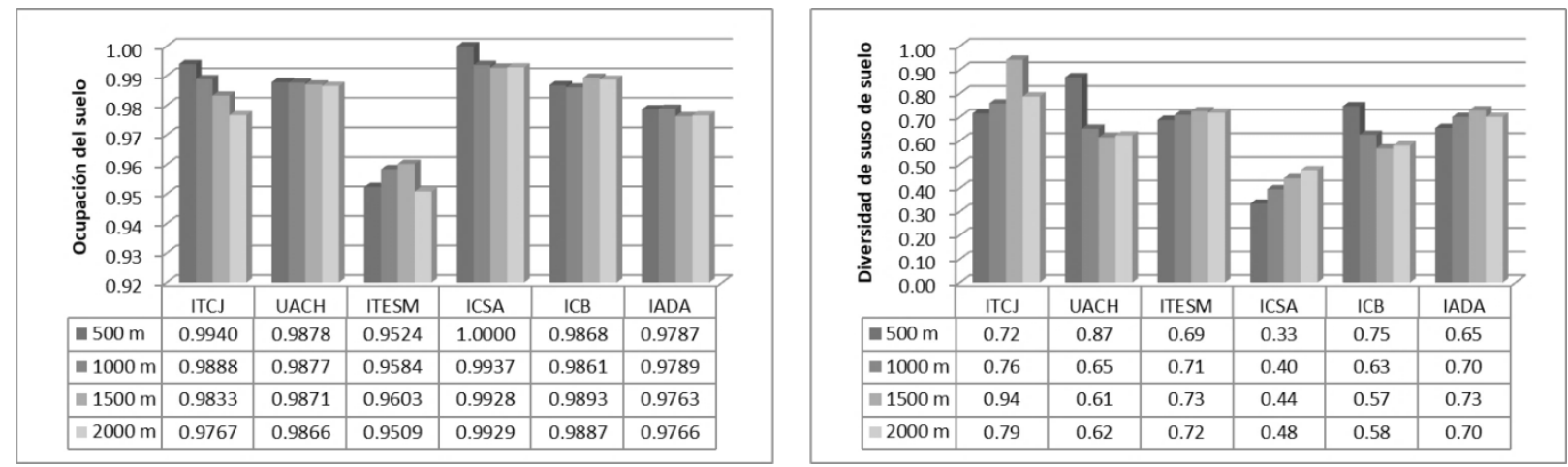

Fuente: los autores. 


\section{Accesibilidad}

El campus de ICSA es el único que coincide con un hot spot de accesibilidad a transporte público, pudiendo ser indicio de la falta de opciones de movilidad distinta al automóvil en la ciudad. La aglomeración de valores altos para accesibilidad a vialidades principales coincide con UACH, ICSA, ICB e ITESM. Ningún campus coincide con hot spots o cold spots de accesibilidad a subcentros (Fig. 7).

Figura 7. Aplicación del índice $\mathrm{Gi}^{*}$ de Getis-Ord para la esfera de accesibilidad.
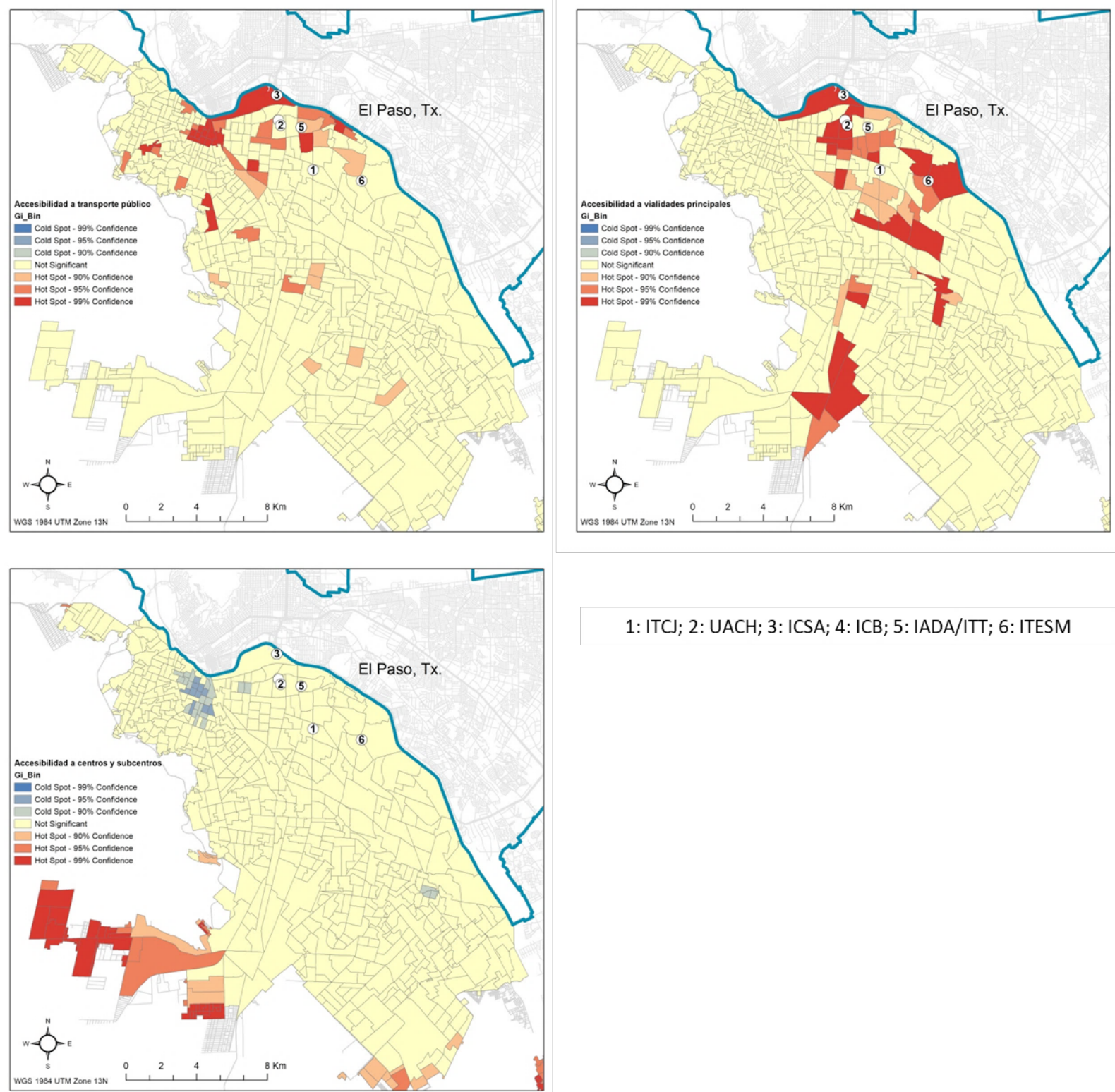

1: ITCJ; 2: UACH; 3: ICSA; 4: ICB; 5: IADA/ITT; 6: ITESM

Fuente: los autores.

Los nodos entre rutas de transporte público son mayores a menor distancia en el caso de ICSA y IADA-ITT. Los nodos de vialidades principales aumentan en cercanía a ICSA, lo mismo ocurre en ITESM pero con menor diferencia. El resto de los casos muestra 
mayor número de nodos entre vialidades principales a mayor distancia de su localización. ITCJ, UACH, ICB IADA-ITT tienen mayor cercanía a un centro o subcentro. Mientras que ITESM e ICSA se encuentran más alejados a uno de ellos (Fig. 8).

Figura 8. Variables de la esfera de accesibilidad a distintas bandas de distancia.
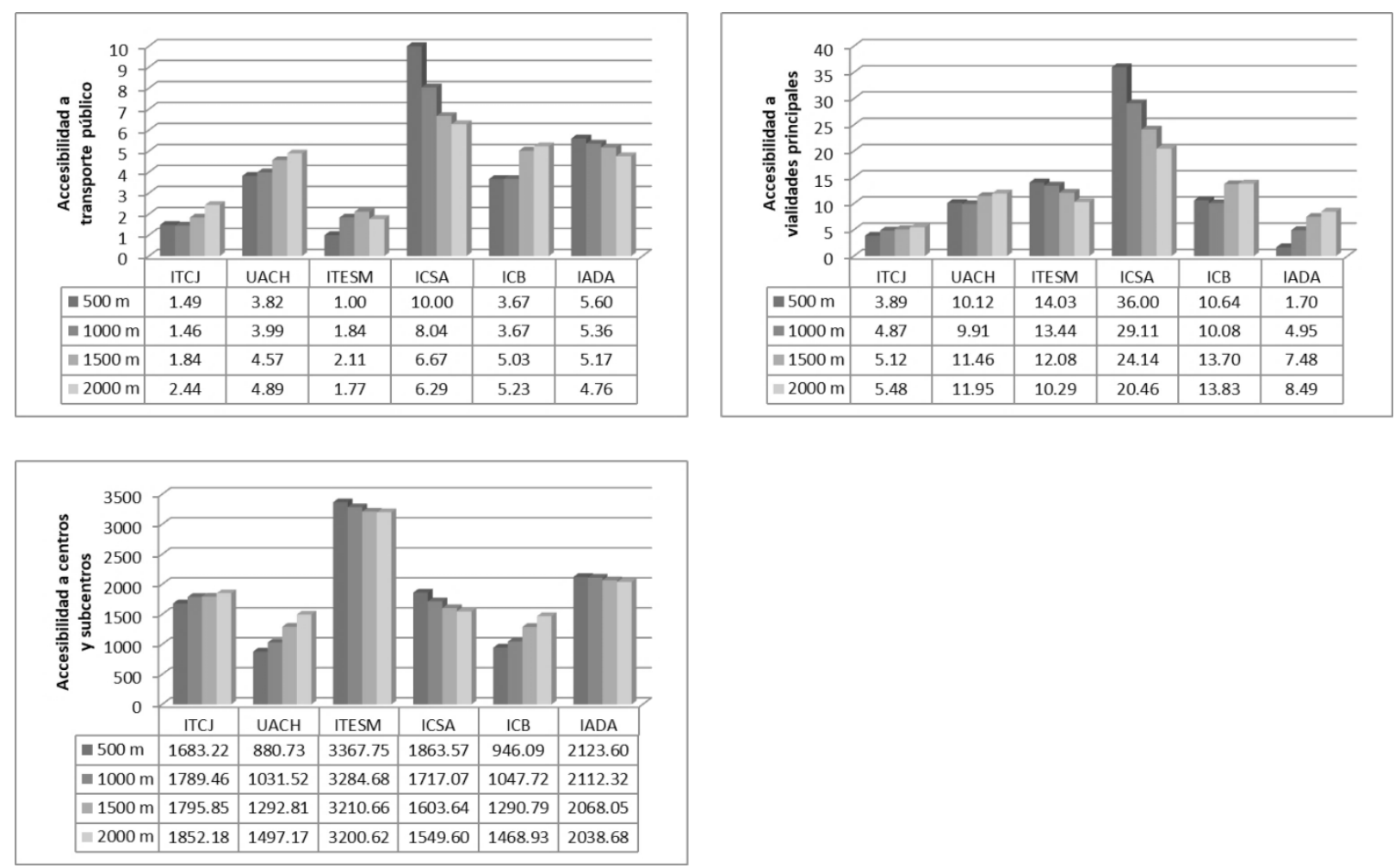

Fuente: los autores.

\section{Conclusión}

Los resultados del análisis espacial en Ciudad Juárez no definen a ningún campus como configurador urbano de manera concisa. Ninguno de ellos coincidió con hot spots ni presentó mayor influencia a menor distancia en la mayoría de las variables. La concentración económica o la accesibilidad al transporte público podrían considerarse primeras manifestaciones de impacto, ya que reflejan servicios que suelen aumentar en cuanto existe mayor demanda causada por la presencia de estudiantes pero tampoco parecen aumentar de manera significativa.

La revisión bibliográfica muestra diversas formas en que la locación de una IES puede contribuir al entorno urbano. Sin embargo, la metodología para poder evaluar la existencia de dicho impacto en el contexto mexicano no ha sido explorada a fondo. Se han expuesto las ventajas y limitantes de una perspectiva cuantitativa. Si bien se perderán las particularidades también existirá el beneficio de excluir la subjetividad proveniente de los actores implicados en los resultados obtenidos. Esta primera propuesta no incluye, de forma implícita, recomendaciones para potencializar beneficios y controlar afectaciones provenientes de la localización de una IES, pero pretende contribuir a la reflexión sobre la 
necesidad de políticas públicas que exploren a este tipo de instituciones como elementos urbanos partícipes en la configuración.

\section{REFERENCIAS}

BENNEWORTH, Paul; CUNHA, Jorge. Universities contributions to social innovation: reflections in theory \& practice. European Journal of Innovation Management, v. 18, n. 34, p. 508-527, 2015.

BENNEWORTH, Paul; CHARLES, David; MADANIPOUR, Ali. Building Localised Interactions between Universities and Cities. European Planning Studies, v.18, n. 10, p. 1611-1629, 2010.

CONAPO. Catálogo Sistema Urbano Nacional 2012. México: Consejo Nacional de Población, 2012.

DEN HEIJER, Alexandra. Managing the University Campus in an Urban Perspective: Theory, Challenges and Lessons from Dutch Practice. Corporations and Cities, 2008. Disponible en: http://medewerkers. tudelft.nl/fileadmin/Faculteit/BK/Actueel/Symposia_en_congressen/CRE_2008/Papers/doc/ Paper02_denHeijer.pdf. Aceso en: 20 ago. 2017.

FERNÁNDEZ-ESQUINAS, Fernando; PINTO, Hugo. The Role of Universities in Urban Regeneration: Reframing the Analytical Approach. European Planning Studies, v. 22, n. 7, p. 1462-1483, 2014.

GARCÍA, José Luis; GARCÍA, Francisco Miguel. Análisis espacial de la complejidad del sistema urbano como soporte de una planificación y gestión urbana sostenibles. En CONESA, Carmelo; ÁLVAREZ Yolanda; GRANELL, María del Carmen. El empleo de los SIG y la Teledetección en Planificación Territorial. Murcia: Universidad de Murcia, 2005.

GETIS, Arthur; ORD; Keith. The Analysis of Spatial Association by Use of Distance Statistics. Geographical Analysis, v.24, n.32, p. 189-206, 1992.

HAINING, Robert. Spatial data analysis: scientific and policy context. Cambrigde: Cambridge University Press, 2003.

HUBBARD, Rob; KITCHIN, Rob; VALENTINE, Gil. Key Thinkers on Space and Place. Londres: SAGE, 2008.

JULIANI, Douglas; TAMILIS DA SILVA, Ania; CUNHA, Jorge; BENNEWORTH; Paul. Universities' Contributions to Sustainable Development's Social Challenge: A Case Study of a Social Innovation Practice. International Journal of Social Ecology and Sustainable Development, v. 8, n. 3, p. 1-18. DOI: 10.4018/ IJSESD.2017070101

LOREY, David. El sistema universitario y el desarrollo económico en México desde 1929. Revista de la Educación Superior, n.89, p. 23-35, 1984.

McGARIGAL, K.; MARKS, B.J. Fragstats: spatial pattern analysis program for quantifying landscape structure. Portland: Pacific Northwest Research Station, 1995.

PEET, Richard. Modern Geographial Thought. Malden: Blackwell Publishing, 1998.

RUSSO, Antonio Paolo; VAN DEN BERG, Leo; LAVANGA, Mariangela. The Student City: Strategic Planning for Student Communities in EU cities. Rotterdam: Ashgate, 2004.

Data de submissão: 07/set./2017

Data de aceite: $24 /$ jan./2018 